\title{
Embolotherapy for massive upper gastrointestinal haemorrhage secondary to metastatic renal cell carcinoma: report of three cases
}

\author{
M A Blake, A Owens, D P O’Donoghue, D P MacErlean
}

\begin{abstract}
Three cases are reported in which angiographic embolisation therapy was effective in arresting upper gastrointestinal bleeding secondary to metastatic hypernephroma. Each patient had histologically confirmed disseminated renal cell carcinoma and presented with recurrent haematemeses with a successful outcome following palliative embolisation therapy. (Gut 1995; 37: 835-837)
\end{abstract}

Keywords: embolotherapy, gastrointestinal haemorrhage, hypernephroma, metastases.

Patients suffering from recurrent haematemeses caused by metastatic disease may pose difficult treatment dilemmas for their doctors. It has been established that in many cases arterial embolisation may be an acceptable and effective way of limiting metastatic gastrointestinal haemorrhage. There are few reports of this technique being applied to bleeding originating from disseminated renal cell carcinoma and we present three such cases in which embolisation played a useful part in controlling upper gastrointestinal haemorrhage.

\section{Case reports}

Case 1

A 63 year old man presented with recurrent severe haematemeses. A right nephrectomy had been performed six years previously for

Departments of Radiology M A Blake A Owens D P MacErlean

and Gastroenterology D P O'Donoghue

St Vincents Hospital, Dublin, Ireland

Correspondence to: Dr M A Blake, Department of Diagnostic Imaging, $\mathrm{St}$ Vincent's Hospital, Elm Park, Dublin 4, Ireland. Accepted for publication 29 March 1995

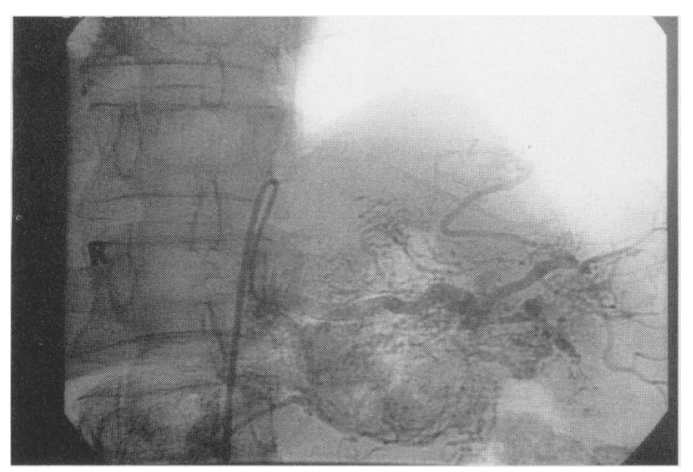

Figure 1: Hypervascular tumour supplied from branches of splenic artery. renal cell carcinoma and a subsequent lobectomy for pulmonary metastases. Endoscopy and biopsy showed invasion of the gastric mucosa by metastatic neoplasm.

Coeliac angiography showed a widespread hypervascular tumour in the gastric and duodenal area with multiple feeding vessels from branches of the splenic artery (Fig 1). Following subtotal occlusion of the splenic artery with gel-foam embolisation (Fig 2) bleeding stopped for three months. Further gel-foam embolisation of the splenic artery was then successfully carried out on recurrent tumour vessels in the gastric area (Fig 3). No complications occurred during either procedure and at five month follow up no pancreatic ill effects were noted and no further episodes of upper gastrointestinal haemorrhage had occurred.

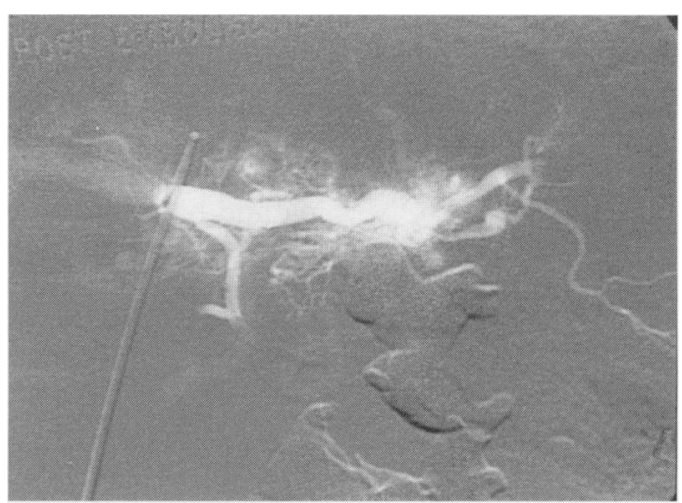

Figure 2: Postembolisation appearance with subtotal occlusion of splenic artery. Contrast in calyceal system of left kidney.

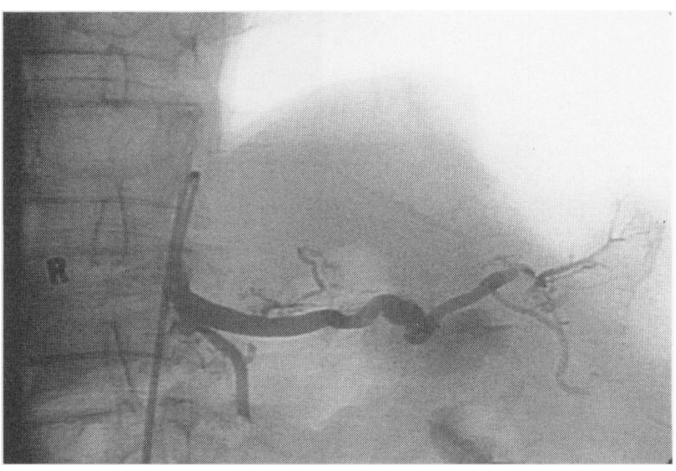

Figure 3: Postembolisation of splenic artery with gel-foam fragments. Contrast in calyceal system of left kidney. 


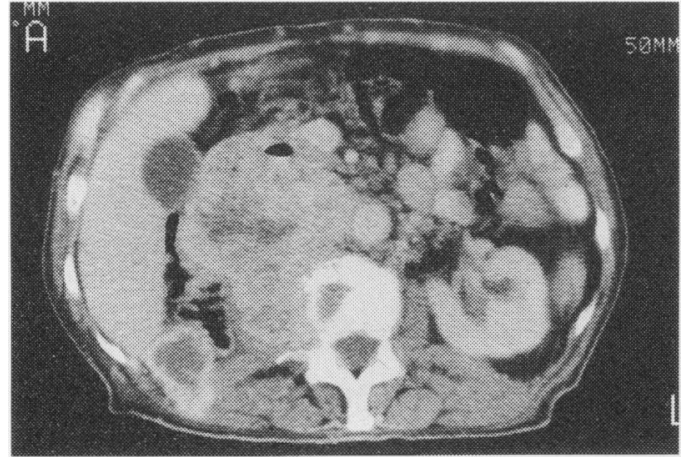

Figure 4: Computed tomography showing huge tumour mass arising from right renal bed and invading the duodenum and right psoas muscle with a second vascular nidus lying posterolaterally.

\section{Case 2}

A 73 year old man was admitted after a severe upper gastrointestinal bleed with haematemesis. A radical nephrectomy had been performed two years before for renal cell carcinoma and subsequently a lobectomy for a hepatic metastasis. Endoscopy showed a metastatic tumour in the descending duodenum. Dynamic computed tomography showed a large mass arising from the renal bed invading the psoas muscle and duodenum (Fig 4). Coeliac angiography showed that the gastroduodenal artery supplied the tumour mass with extravasation of contrast (Fig 5). Embolisation of the gastroduodenal artery with gel-foam fragments was performed (Fig 6). The procedure was well tolerated and at follow up no pancreatic complications were noted and no further bleeding problems were evident.

\section{Case 3}

A 78 year old man with confirmed inoperable disseminated renal cell carcinoma was referred for palliative embolisation. He had presented with chronic intractable gastrointestinal bleeding and haematuria. Investigations had shown invasion of the duodenum by the hypernephroma and haemorrhage was controlled by embolisation of the right renal and gastroduodenal arteries with gel-foam particles. No

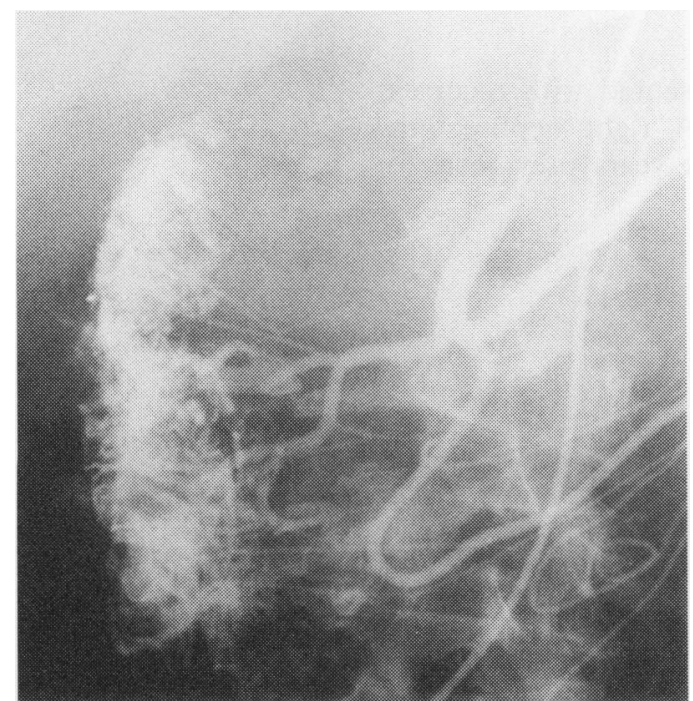

Figure 5: Angiogram showing tumour circulation arising primarily from gastroduodenal artery.

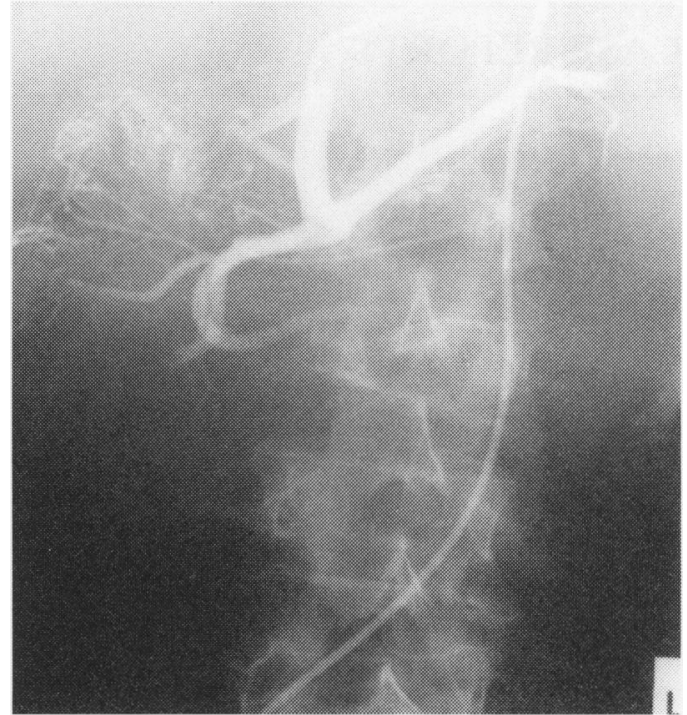

Figure 6: Appearance after embolisation of gastroduodenal artery with occlusion of duodenal, pancreatic, and gastroepiploic branches.

further episodes of bleeding were recorded but he died some weeks later from his terminal disease.

\section{Discussion}

Metastasis to the upper gastrointestinal tract from renal cell carcinoma manifesting with haematemesis is rare. ${ }^{1} \mathrm{~A}$ review of published works shows two reports of embolisation for control of upper gastrointestinal haemorrhage arising from metastatic renal cell carcinoma. ${ }^{23}$

Our patients had their diagnoses made and confirmed with complementary endoscopy and coeliac angiography. Embolisation of tumour feeding vessels was performed with plugs of gelatin sponge. Each patient tolerated the procedure well and did not show any symptoms of tumour necrosis or bowel infarction. Two were able to leave the hospital soon after the embolisation and did not experience rebleeding after completed embolisation. The third terminally ill patient had a satisfactory response to embolisation therapy but succumbed soon after to metastatic effects unrelated to the procedure.

All were elderly patients with disseminated malignant disease, terrified at the prospect of possible exsanguination, who, before the embolisation were confined to hospital with recurrent severe haematemeses. The patients and their families, however, found the procedure comforting and worthwhile and two of the patients were able to return to their homes.

Until recent years major surgery or frequently ineffective medical treatment were the options available to treat metastatic upper gastrointestinal bleeding. Transcatheter arterial embolisation has been successfully applied to a wide range of clinical situations ${ }^{4}$ and it is now established that selective embolisation of the offending feeding artery may often be the treatment of choice in metastatic gastrointestinal bleeding.

Haematemesis resulting from metastatic renal cell carcinoma is a rare but distressing event. We here describe three cases of this 
phenomenon treated by transcatheter embolisation. In our experience angiographic embolisation is useful in the treatment of these patients by arresting the otherwise inexorable progress of metastatic gastrointestinal haemorrhage.

1 Graham AP. Malignancy of the kidney: survey of 195 cases. fUrol 1947; 58: 10-21.
2 Lesch-Nyhan A, Fishman EK, Kadir S. Diagnosis and management of massive gastrointestinal bleeding owing to duodenal metastasis from renal cell carcinoma. $\mathcal{f}$ Urol 1987; 138: 611-3.

3 Gordon B, Lossef SV, Jelinger E, Barth KH. Embolotherapy for small bowel haemorrhage from metastatic renal cell carfor small bowel haemorrhage from metastatic renal cell carcinoma: case report. Cardiovasc Intervent Radiol 1991; 14: $311-3$

4 O'Halpin O, Legge D, MacErlean DP. Therapeutic arterial embolization: report of 5 years experience. Clin Radio 1984; 35: 85-93 